\title{
CALCULATING TARGET THRESHOLDS FOR THE MARGIN VALUE METHOD USING COMPUTATIONAL TOOLS
}

\author{
A. Brahma ${ }^{\otimes}$ and D. C. Wynn \\ The University of Auckland, New Zealand \\ $\square$ abra248@aucklanduni.ac.nz
}

\section{Abstract}

Overspecification or excess margin in a design can enhance its ability to absorb changes and uncertainty, but also deteriorates performance criteria such as weight and cost. This paper shows how the Margin Value Method (article in review) can be applied in conjunction with CAE tools such as FEA to quantify excess margin where a design is too complex for algebraic analysis. This new application context for the MVM is illustrated using a case study of a flange coupling design, in which topology optimisation is used within the MVM to identify opportunities for design improvement.

Keywords: margin, design optimisation, margin value method (MVM), engineering change, computational design methods

\section{Introduction}

Overspecification of elements of an engineering design can lead to creation of excesses called margins. Margin in design can influence important metrics such as cost, safety and changeability, and therefore should be managed carefully. In practice, however, margins are often handled heuristically or "in a crude quantitative manner" that varies depending on the individual and organisation, with "little or no rigorous method". Consequently, overuse or underuse of margins can have contrasting effects on these metrics. For instance, insufficient margin can result in substantial design rework, delayed and overbudget projects, and even potential design failures. On the other hand, excessive use of margin can lead to the product being overdesigned and less competitive in terms of cost. Margins are often used by engineers to help to mitigate the risks and uncertainties inherent to product development (Tackett et al., 2014), which occur throughout the lifecycle of a product. These uncertainties can be associated with requirements (Watson et al., 2016), analysis models used during design (Guenov et al., 2018), manufacturing variability (Zhu and Ting, 2000; Morse et al., 2018) and product operation. Margins also play an important role in determining whether a change will propagate or not, by either absorbing a change or letting it propagate further. On the one hand margins could make a design more robust to changes and uncertainties, the overspecification also often entails design performance loss. In practice it can be difficult to locate margins in a design and to size them depending on the desirability of its impacts. Even if located and sized, margins could be difficult to eliminate due to constraints exogenous to the design, such as manufacturing methods.

An approach called the Margin Value Method (MVM) has been developed to identify, localise and size excess margin in a design, considering margins which can be used up to mitigate future changes (Brahma 
and Wynn, 2019). The present paper extends the MVM for application to more complex design situations. In particular, Brahma and Wynn (2019) illustrate the MVM using a case of bulk material conveyor system whose design could be completed by decisions combined with evaluating fundamental engineering relations expressed as algebraic equations. They do not address more complex situations where computational methods such as Finite Element Analysis (FEA) must be used to complete the design. This paper, therefore, explores application of MVM in such a case and discusses implications and further work opportunities. To illustrate the enhanced method a study of a rigid flange coupling design is used.

\section{Literature review}

The importance of margins in design is well recognised by researchers (Eckert et al., 2004), both for absorbing the adverse effect of uncertainty and future changes (Eckert and Isaksson, 2017), as well as for undesirably deteriorating the design performance if too much margin is included. The interest in the topic has increased substantially in recent years. A comprehensive review of such work is recently reported by Brahma and Wynn (2019). This section summarises some key points relevant to the present paper.

Unknown risks are often tackled using margins of different types. A well-known example of margin is factor of safety, referring to use of a multiplication factor to over-design keeping safety and reliability in mind. Many publications focus on determination of such factors, which are context and fieldspecific. For example, Ghosn and Moses (1986) and Fenton et al. (2015) focused on this problem in bridge engineering, Mohammed et al. (2016) focused on ship hull design and so on. A list of suitable factors and influences on the factor of safety, depending on application context, was published by Collins (2010). These factors were further adopted by Iorga et al. (2012) to develop a general-purpose rating system for deterministic estimation of safety factors. These methods for sizing margin are well developed in the context of individual elements of a system, but when it comes to entire designs, their application tends to rely on experience and judgement of engineers and managers.

A number of authors have also proposed approaches and models that consider more broadly how margins can be sized and allocated in engineering design. Authors such as Tilstra et al. (2015), provided guidelines for approaches using margins to make the design more flexible towards future evolutionary changes. Their work was further extended by Tan et al. (2016), who used Multidisciplinary Optimisation (MDO) to trade-off performance and design margins, thereby potentially increasing the robustness of the design. Another model was proposed by Cansler et al. (2016) who used functional decomposition to model margins and quantify them. Guenov et al. (2018) introduced the concept of "explicit margin space", derived from the concept of design space, to study the influence on and limits of allowable margins on certain parameters by allocating margins on certain other parameters. More recently Lebjioui (2018) reported a method in the context of change propagation in design. Lebjioui hypothesises that a large amount of margin can be related to low change propagation probability and applies CPM (Clarkson et al., 2004) to identify components with significant margins.

Another method for analysing margin in design is the Margin Value Method (MVM) of Brahma and Wynn (2019). Because this is the basis of the current paper it is described in greater detail in the next section.

\section{Overview of Margin Value Method (MVM)}

Designers make decisions during a design process, and these decisions are what lead to creation of margins. Examples of margin-creating decisions include selection of off-the-shelf components from a manufacturer's catalogue, or modifying parameters from their ideal values to simplify manufacturing. The MVM is based on pinpointing these decisions and the resulting margin, and is intended to be used where a design exists and is to be improved incrementally by modifying parts one at a time. It first identifies and quantifies margins in the design and then provides guidance towards prioritisation of excess margins for reduction by redesigning parts, considering each margin's desirable ability to absorb potential design changes vs. their undesirable impact on the design's performance. The method also identifies the extent of possible utilisation of margins, keeping in mind opportunities for 
absorbing future changes. The following information provides only a brief summary of the three steps of the method as needed to explain the current paper. The method is presented more comprehensively with some other examples by Brahma and Wynn (2019).

\subsection{Creating a margin analysis network}

A margin analysis network relates the inputs (i.e. specifications) for the design, the performance parameters of the design, the intermediary parameters calculated during the design process and the design decisions. In the method, the network must be created based on an understanding of the design process for the specific design at hand. In the example shown in Figure 1, a clutch is to be selected for an application where the coefficient of friction and actuation force are provided as inputs. Node $f_{1}$ calculates the torque required and is given by $0.5 \mathrm{~F} \mu$. Based on this value a decision is made in which the designer chooses a clutch. Torque transmission capability of the clutch is $\mathrm{T}_{\text {sel }}$; this is an example of a decided value in the method, so-called because it results from a decision. An important aspect of the margin analysis network is the inclusion of margin nodes, which show where the decided values of parameters (determined as outputs from decisions) must satisfy certain threshold values, denoting the minimum of maximum for that decided value to be acceptable. Typically threshold values result from earlier calculations and decisions. For the example, the threshold value relating to the clutch torque is $\mathrm{T}_{\text {req. }}$. The difference between the decided and threshold values $\left(T_{\text {sel }}-T_{\text {req }}\right)$ is called the excess margin (shown as E1 in Figure 1), and represents the amount by which the threshold value can change before the corresponding decision becomes invalid, so it needs to be revisited and the design changed accordingly. The greater the excess margin, the greater the magnitude of change the design may be able to absorb. However, this depends on the position of the margin node in the network and the particular relationship between the threshold value and the input parameter(s) that are subject to change.

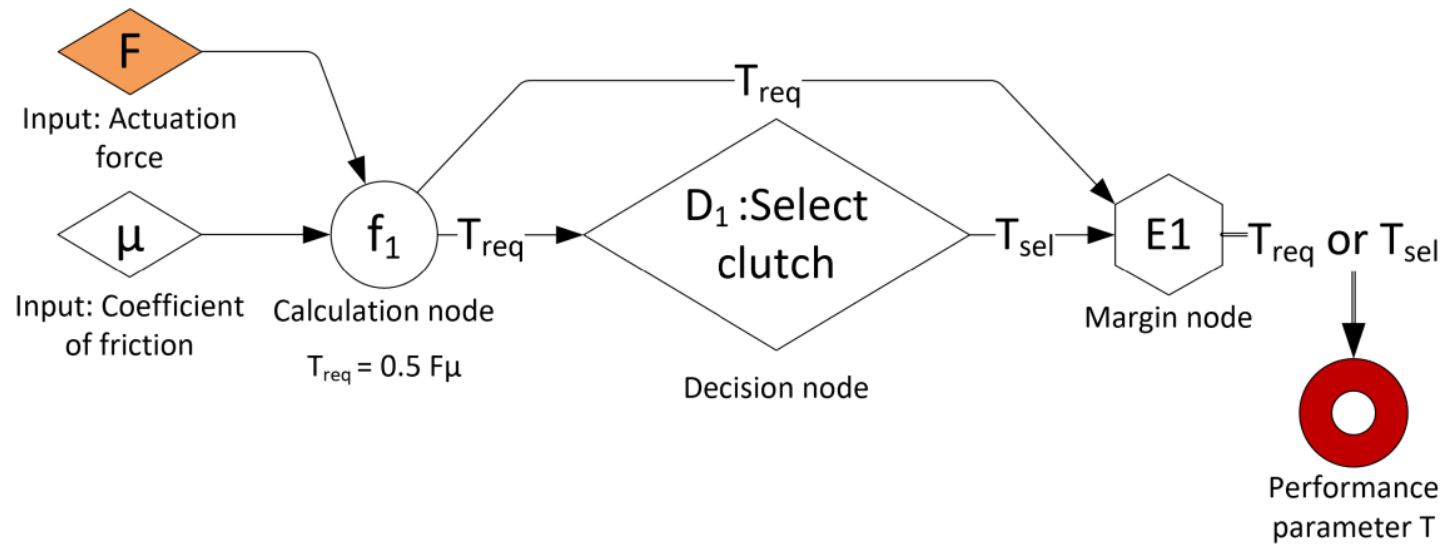

Figure 1. A simplified example for a clutch selection

\subsection{Completing a margin value analysis}

The margin value analysis involves calculation of three metrics for each excess margin in the network described above. Taken together, these metrics indicate the value of each excess margin for the design as a whole.

The first metric quantifies the local excess at each margin node:

$$
\text { Excess }_{m}=\frac{\text { decided }_{m}-\text { threshold }_{m}}{\text { threshold }_{m}}
$$

Where, decided $\mathrm{m}_{\mathrm{m}}$ is the decided value at the input to margin node $\mathrm{m}$, and threshold $\mathrm{m}_{\mathrm{m}}$ is the target threshold at the input to margin node m.

For the example of the clutch,

$$
\text { Excess }_{E 1}=\frac{T_{\text {sel }}-T_{\text {req }}}{T_{\text {req }}}
$$


The second metric is based on the observation that the excess represents overspecification that can be propagated downstream through the network to deteriorate design performance parameters, i.e. $\mathrm{T}$ in the example. Metric 2 captures this undesirable effect and is given by;

$$
\text { Impact }_{m j}=\frac{P_{j\left(\text { decided }_{m}\right)}-P_{j\left(\text { threshold }_{m}\right)}}{P_{j\left(\text { threshold }_{m}\right)}}
$$

Where,

- $P_{j\left(\text { decided }_{m}\right)}$ is the value of performance parameter $\mathrm{j}$ ( $\mathrm{T}$ in the clutch example) when the output of margin node $\mathrm{m}$ (E1 in the example) is set to the decided value that is input to that node, and all dependent downstream values are recalculated.

- $\quad P_{j\left(\text { threshold }_{m}\right)}$ is the value of performance parameter $\mathrm{j}$ when the output of margin node $\mathrm{m}$ is set to the target threshold that is input to that node, and all dependent downstream values are recalculated.

Impact for the clutch therefore can be written as:

$$
\operatorname{Impact}_{E 1 T}=\frac{P_{\left(T_{s e l)}\right.}-P_{\left(\mathrm{T}_{\text {req }}\right)}}{P_{\left(\mathrm{T}_{\text {req }}\right)}}
$$

The third metric calculates the desirable contribution of each margin node in preventing changes to input parameters from causing design changes, as represented by the need to revisit a decision. Essentially, as the input parameter increases, the threshold values in the margin analysis network will also increase, caused indirectly through the network of design calculations. To calculate the metric each input parameter $\mathrm{P}_{\mathrm{i}}$ (Force $\mathrm{F}$ in the case of the clutch example) is increased till the point where any of the threshold values meet the corresponding decided values. The value of this $P_{i}$ is recorded as $\operatorname{Pmax}_{\mathrm{i}} . \mathrm{Pmax}_{\mathrm{i}}$ is an estimate of the maximum value of the input parameter $\mathrm{i}$ that could be supported by the design. Finally this is expressed as a proportion:

$$
\text { Deterioration }_{i}=\frac{\operatorname{Pmax}_{i}-P_{i}}{\operatorname{Pmax}_{i}}
$$

For the example,

$$
\text { Deterioration }_{F}=\frac{\operatorname{Pmax}_{F}-P_{F}}{\operatorname{Pmax}_{F}}
$$

The final step in the metric calculation determines by how much each excess margin in the network is used up to absorbing this maximum deterioration. For each input parameter, the new input value Pmax $_{\mathrm{i}}$ is first substituted in place of the original value. The target threshold for every margin node is then recalculated and written as thresholdnew (For the example, Tnew ${ }_{\text {req }}$ ). The proportion of original margin remaining is then calculated:

$$
\text { Absorption }_{\text {im }}=\frac{\text { thresholdnew }_{\text {im }}-\text { threshold }_{m}}{\text { threshold }_{m} \cdot \text { Deterioration }_{i}}
$$

Absorption for the clutch example is calculated as:

$$
\text { Absorption }_{F E 1}=\frac{\text { Tnew }_{\text {req }}-T_{\text {req }}}{T_{\text {req }} \cdot \text { Deterioration }}
$$

The calculated values of the metrics in Equations (4) and (7) are averaged over the performance parameters $\mathrm{j}$ and input parameters i respectively, allowing each excess margin to be positioned on an $\mathrm{XY}$ plot. Each point on the plot is then shown as a circle, where the bubble sizes are determined by the values calculated in Equation (1). The guidance as shown in Figure 2 is then used to interpret the data. For more detail and more examples, the reader may refer to Brahma and Wynn (2019). 


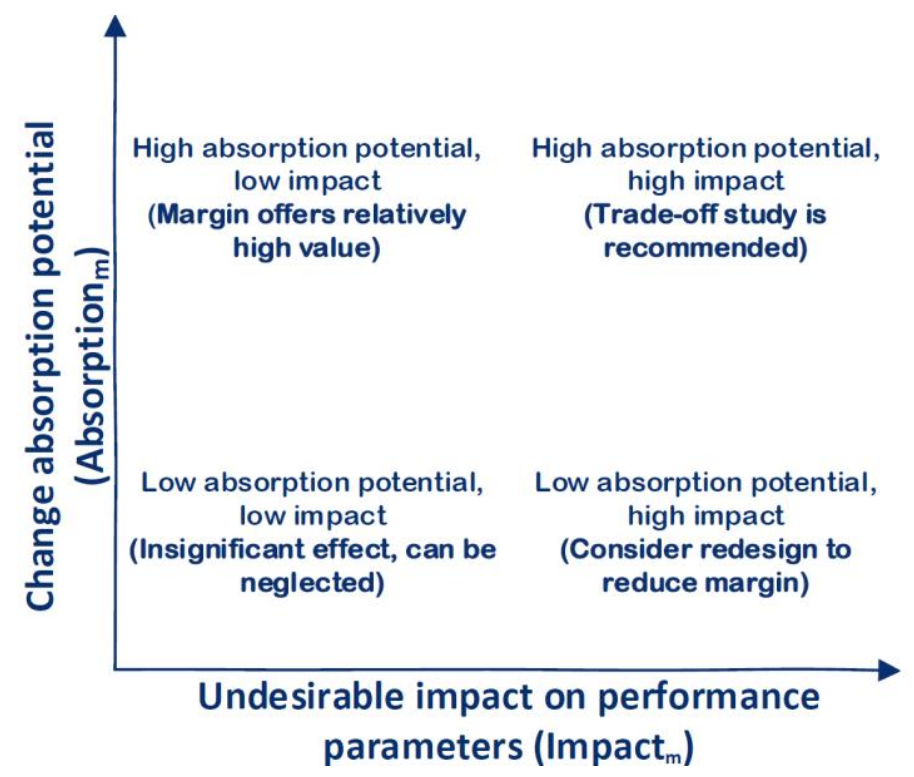

Figure 2. Margin Value Plot showing four regions and their interpretation (Brahma and Wynn, 2019)

\subsection{Contribution of this paper}

The case study reported in Brahma and Wynn (2019) to illustrate the method was a conveyor design, which could be completed using simple calculations on a hand calculator. In this paper the approach is extended to show how it can be applied to situations in which not all parameters may be expressed through mathematical relationships, such that the designer must use computational methods such as finite element analysis. Application of the MVM in this context is explained in the next section.

\section{Application of MVM to a design situation requiring FEA}

Figure 3 (left) is a representative image of a shaft coupling, used to illustrate the new contribution of this paper. The flange coupling is of a protected type which can be used to transmit a torque of 17800 $\mathrm{Nm}$, from one rotating shaft to another. The coupling is mounted on the shafts through keys and is held together using hex nuts and bolts. Input parameters (specifications) include a maximum allowable shear stress of $125 \mathrm{~N} / \mathrm{mm}^{2}$. While the MVM allows for any number of input and performance parameters, this case study uses only one selected input and one performance parameter so that the focus is kept on the consideration of FEM in the MVM process.
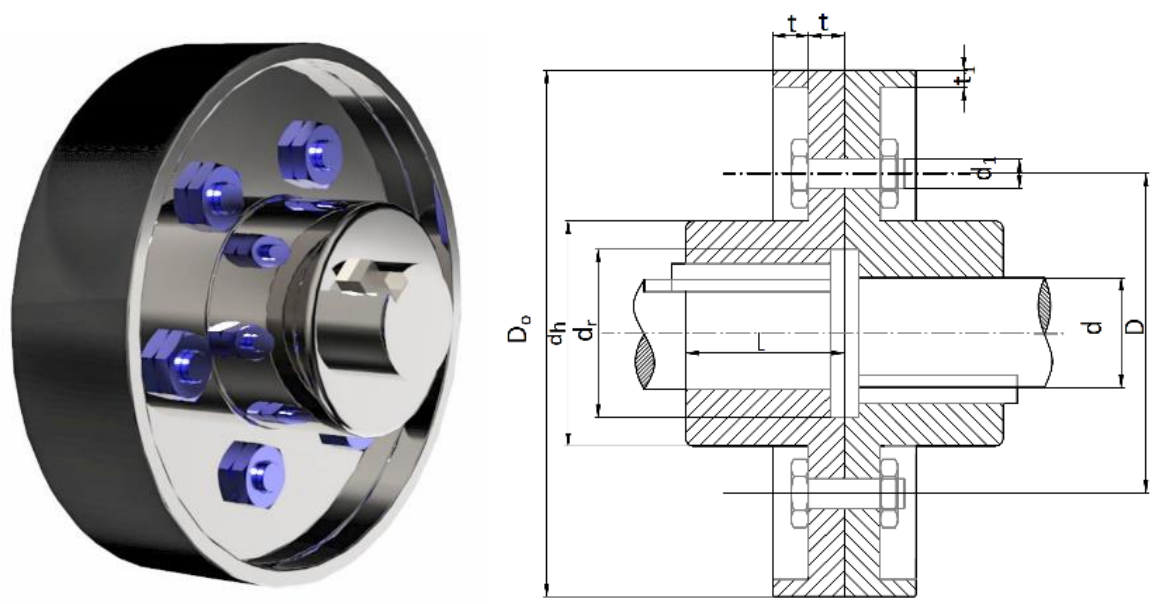

Figure 3. (Left) A flanged coupling of protected type to transmit torque between two collinear shafts, connected to the hub using rectangular keys. Uses six hex nuts and bolts to join the flanges together. (Right) Proportions of rigid coupling as per guidelines of Bhandari (2010) 


\subsection{Creating a margin analysis network of the coupling}

The design process model as shown in Figure 4 was developed from standard practices and procedures as stipulated by machine design textbooks such as Bhandari (2010) and Budynas and Nisbett (2014). The design process consists of 4 sub-tasks starting with the design of shaft. The second task involves the design of the protected flanged hub followed by selection of standard hex nuts and bolts and selection of standard keys to transmit the required torque. Further, a performance parameter reflects the weight of the entire assembly, at the right-hand side of the diagram.

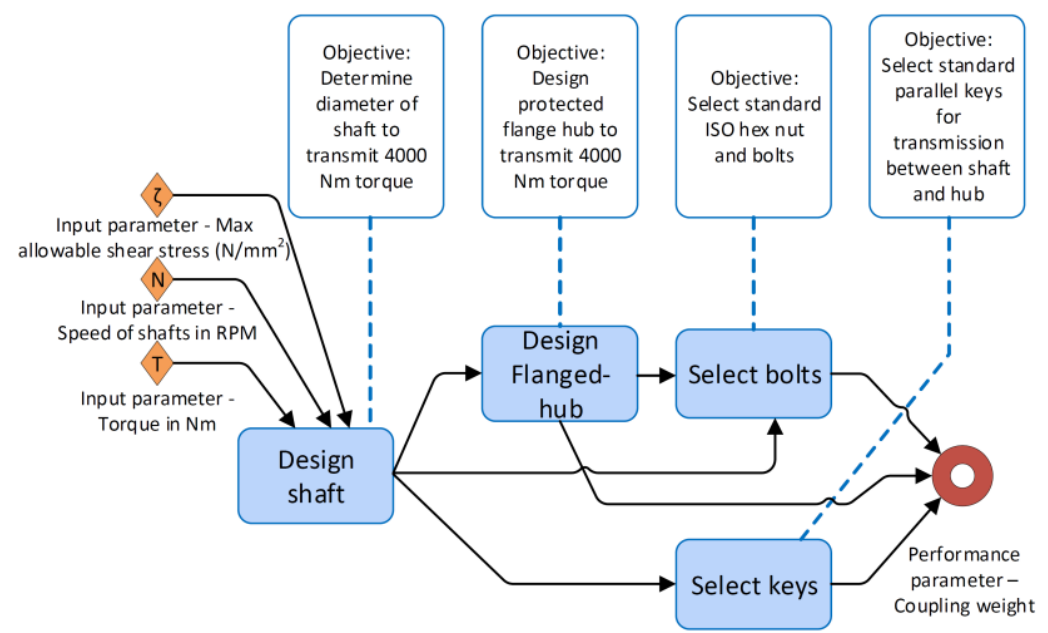

Figure 4. Design process model of a protected flange coupling. Figure shows input parameters, design steps, objectives and performance parameters

In the next step, each task was systematically considered to identify the parameters it requires and produces, and these were connected to form the Margin Analysis Network shown in Figure 5. Node 1 for example, is a calculation step used to calculate the shaft diameter and can be written as shown in Equation (9) based on basic engineering principles:

$$
d=\sqrt[3]{\frac{16 T}{\pi \tau}}
$$

Where $\mathrm{T}$ is the torque required to be transmitted and $\tau$ is the allowable shear stress of the material to be used. For this case study, the calculated shaft diameter d was $90 \mathrm{~mm}$.

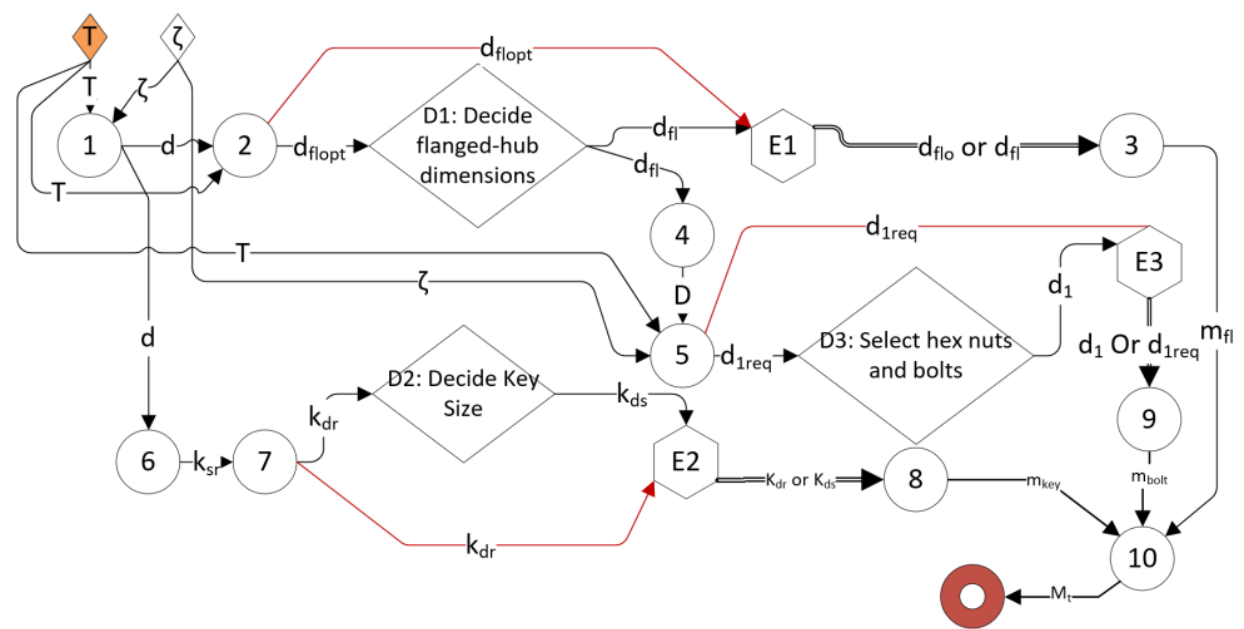

Figure 5. Margin analysis network for the coupling design

Node 2, represents calculation of the minimum required dimensions of the flanged hub to prevent failure, which is given by $d_{\text {flopt. }}$ Guidelines to design a flanged-hub are given by empirical relationships 
based on the shaft diameter developed by engineers over the years (Bhandari, 2010). For example, the thickness of the flange (Figure 3 - Right) is given as $t=0.25 \mathrm{D}+7.50 \mathrm{~mm}$, where $\mathrm{D}$ is the shaft diameter in $\mathrm{mm}$. These empirical relationships or standard proportions, however, cannot be verified through algebraic methods because of the complex geometry. Since they are empirically determined, they are over-conservative and do not represent the true minimum required value. This causes a problem in computing the threshold value relating to the node E1. E1 here is the margin node at which the margin relating to the flange dimensions is calculated. Similarly, E2 and E3 are the margin nodes relating to the key size and the fasteners respectively.

If the formula described above is used to calculate the threshold value, the MVM will underestimate the actual margin in the coupling dimensions and therefore would not indicate the full theoretical improvement that might be possible for the design by redesigning the coupling. To resolve this problem and hence extend the MVM for more realistic cases where threshold values cannot be calculated analytically from physical principles, finite element method was used to develop the minimum required dimensions of the flanged hub based on optimisation of basic geometry. A massbased topology optimisation algorithm was run in ANSYS to more closely estimate the least possible mass required to sustain the applied torque, and hence produce a more accurate threshold value for the MVM. Topology optimisation is chosen specifically as the performance parameter relates to the overall mass of the coupling. In case the performance is measured based on the stress/strain capability of the coupling, other appropriate analysis techniques may be used.

The output of Node 2 therefore, is the minimum required dimensions of the flanged hub to prevent failure and is given by $d_{\text {flopt }}$. The corresponding optimised geometry is shown in Figure 6. However, due to manufacturing constraints, it is not possible to use this geometry for practical purposes. Furthermore, flange protection is necessary from a safety point of view and therefore cannot be removed. In decision node D1, the dimensions were decided which considers all these aspects along with usability, based aspects such spanner clearance etc. The decided dimensions are $\mathrm{d}_{\mathrm{fl}}$. Both $\mathrm{d}_{\text {flopt }}$ and $\mathrm{d}_{\mathrm{fl}}$ feed into margin node $\mathrm{E} 1$ and then into node 3 which calculates the mass based on the two options. Mass-based on $\mathrm{d}_{\text {flopt }}$ was found to be $12.857 \mathrm{~kg}$ whereas, decided dimensions give mass as $46.616 \mathrm{~kg}$ for one of the parts of the flanged hub.

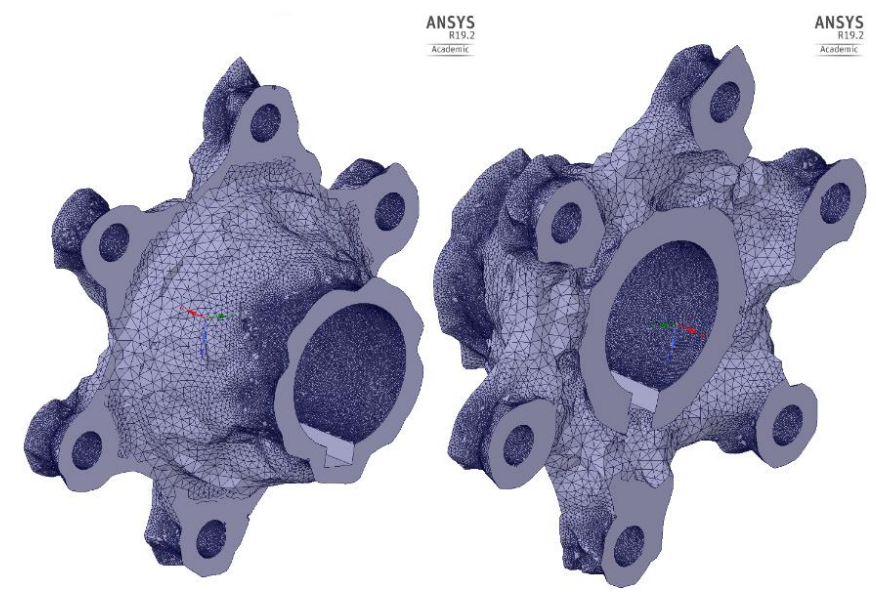

Figure 6. Optimised topology of flanged hub. Figure shows one half of the coupling from two different views

Dimensions for the bolts and keys were calculated using Equations (10) and (11). Minimum required bolt diameter was found to be $15 \mathrm{~mm}$ whereas, minimum dimensions of a key required was found to be of $24 \mathrm{~mm}$ depth. Considering these minima, the selected bolt was ISO M24 (D3) in size whereas, a $28 \mathrm{~mm}$ key was selected in D2.

$$
\begin{aligned}
& d_{1}^{2}=\frac{8 T}{\pi D N \tau} \\
& \tau=\frac{2 T}{d b l}
\end{aligned}
$$




\subsection{Margin value analysis of the coupling}

The procedure outlined in Section 3 was used to calculate the metrics 1 to 3 for the original, simple coupling design shown in Figure 3.

\subsubsection{Metric 1: Local excess margin}

The excess is:

$$
\text { Excess }=\left[\begin{array}{l}
2.646 \\
0.167 \\
3.096
\end{array}\right]
$$

The values indicate that margin at $\mathrm{E} 3$, which relates to the nuts and bolts, is the most highly overspecified at 3.096 times (309.6\%), whereas the margin for the keys are least overspecified at $16.7 \%$. The flanged hub comes close at second with 2.646 times overspecification. The metric values here were calculated keeping all the parts in mind, i.e. E2 considers two keys and E3 considers six nuts and six bolts together.

\subsubsection{Metric 2: Adverse impact of excess margin}

The second metric is used to determine the adverse impact of each of the three margins in Figure 5, on the only performance parameter of the total mass of the system. Although the figure shows two inputs, one being the shear strength of the material, $\tau$ and the other one being the torque being transmitted, T. Since it is unlikely that the material will be changed, only $\mathrm{T}$ is considered as the selected input for analysis.

For the flanged coupling, the resulting impact matrix is shown below;

$$
\text { Impact }=\left[\begin{array}{l}
2.223 \\
0.001 \\
0.031
\end{array}\right]
$$

The rows of the impact matrix in Equation (13) relates to the three margins at E1, E2 and E3 respectively and the column represents the performance parameter $\mathrm{M}_{\mathrm{t}}$. The matrix shows a $222.3 \%$ deterioration of the performance parameter $\mathrm{M}_{\mathrm{t}}$ due to the margin at $\mathrm{E} 1$ (flanged hub) whereas, just $0.1 \%$ deterioration due to $\mathrm{E} 2$ which relates to the key. The nuts and bolts cause a deterioration of performance parameter by $3.1 \%$.

\subsubsection{Metric 3: Benefit of margins for absorbing change}

The likely source of change in this case study was the only input, which is the torque applied on the coupling. It is possible that the coupling may need to be used beyond its capacity to handle torque. The margins in the system may enable such use beyond the rated capability. The metric was calculated in accordance with the procedure mentioned previously. The performance parameter was set at its original value of $98.09 \mathrm{~kg}$. All decision steps were bypassed and all nodes were recalculated using the target thresholds instead of the decided values. The least value of input $\mathrm{T}$, satisfying the network was found to be $19452 \mathrm{Nm}$. Considering this value, the maximum deterioration that can be absorbed in the system is calculated:

$$
\begin{aligned}
& \text { Deterioration }_{i}=[0.093] \\
& \text { Absorption }_{\text {im }}=\left[\begin{array}{l}
0.078 \\
0.896 \\
0.000
\end{array}\right]^{T}
\end{aligned}
$$

\subsubsection{Margin Value plot}

The metrics were used to create the margin value plot of Figure 7. 


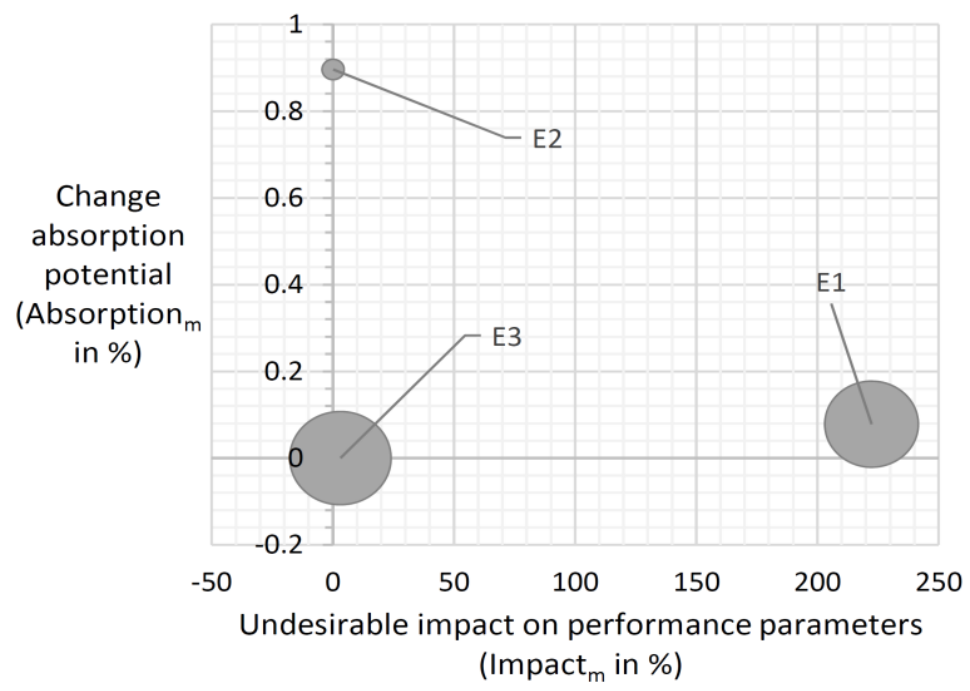

Figure 7. Margin Value Plot for the coupling case, showing all the three metrics on the same visualisation. The radius of each margin indicates the local excess margin. The radii show only relative excess and are not on the scale as the axes

The plot shows the three margins E1, E2 and E3 and the three associated metrics for each one; Absorption, Impact and Excess. The plot shows that E2, which relates to the keys, has a high absorption rate but a low impact on the overall weight of the system. E1, on the other hand, has high undesirable impact on the total weight. E3 has insignificant effect on both absorption and impact metrics. Interpreting the plot on the basis of Figure 2 reveals that E2 provides value for change absorption, whereas E1 should be considered for reduction by redesign. In practical terms this confirms that the component which should be highest priority for optimisation in the coupling is the hub. In this case the result is unsurprising but in designs with larger number of components, the method is helpful to prioritise opportunities for improvement.

\subsection{Summary of additional contributions and challenges}

While the MVM itself is discussed in detail by Brahma and Wynn (2019), this paper adds insight by showing how it can be applied in situations where necessary threshold values cannot be accurately expressed using algebraic expressions. It also suggests how the MVM could help to identify opportunities to improve design performance using additive manufacturing of selected parts. The case study used a topology optimisation method to theoretically eliminate margins which, with traditional manufacturing methods, may not be possible. Methods such as additive manufacturing, however, may be used to create complex topologies thereby more closely approaching the ideal part and improving the overall performance of the system. The margin value method may also offer opportunities to calculate the value of additive manufacturing to improve an existing design, where only certain parts could be optimised and produced in this way for time and cost reasons.

One of the main challenges in applying the MVM in this context was the complex nature of geometry created by the optimisation algorithm. Such complex geometries are difficult to process and are computationally intensive. Since the MVM calls for multiple calculations on the same nodes, these simulations may need to be run multiple times on the same object. Further, so far the method does not support consideration of errors and uncertainties. For example, if margin presented in such analysis is small, it might be difficult to predict how much of the margin is attributed to error associated with the mesh size and/or computation.

\section{Conclusion}

This paper shows how the MVM of Brahma and Wynn (2019) can be applied to designs where computational tools must be used instead of fundamental calculations and algebraic expressions. Although the illustrative example shows how MVM can be adapted for such cases, the chosen case is simple with a relatively small number of parts. In future, more complex designs can be analysed where 
opportunity to optimise more number of parts simultaneously may exist. Further, the current illustration assumes that the optimised part can be manufactured without taking concepts such as Design for $\mathrm{X}$ into consideration. In future, such assumptions are aimed to be relaxed by considering such exogenous constraints on the (re)design and optimisation process. Consideration of design based on alternate methods of embodiment such as additive manufacturing is also an avenue for future work.

\section{References}

Bhandari, V.B. (2010), Design of Machine Elements, 3rd ed., Tata McGraw-Hill, India.

Brahma, A. and Wynn, D.C. (2019), Margin value method for engineering design improvement.

Budynas, R.G. and Nisbett, J.K. (2014), Shigley's Mechanical Engineering Design, McGraw-Hill Higher Education, NY.

Cansler, E.Z. et al. (2016), "Excess Identification and Mapping in Engineered Systems", Journal of Mechanical Design, Vol. 138 No. 8, p. 081103. https://doi.org/10.1115/1.4033884

Clarkson, P.J., Simons, C. and Eckert, C. (2004), "Predicting Change Propagation in Complex Design", Journal of Mechanical Design, Vol. 126 No. 5, pp. 788-797.

Collins, J.A. (2010), Mechanical design of machine elements and machines: a failure prevention perspective, 2nd ed., Wiley, Hoboken, NJ. https://doi.org/10.1115/1.1635406

Eckert, C. and Isaksson, O. (2017), "Safety Margins and Design Margins: A Differentiation between Interconnected Concepts", Procedia CIRP, Vol. 60, pp. 267-272. https://doi.org/10.1016/j.procir.2017.03.140

Eckert, C., Clarkson, P.J. and Zanker, W. (2004), "Change and customisation in complex engineering domains", Research in Engineering Design, Vol. 15 No. 1, pp. 1-21. https://doi.org/10.1007/s00163-003-0031-7

Fenton, G.A. et al. (2015), "Reliability-based geotechnical design in 2014 Canadian highway bridge design code", Canadian Geotechnical Journal, Vol. 53 No. 2, pp. 236-251. https://doi.org/10.1139/cgj-2015-0158

Ghosn, M. and Moses, F. (1986), "Reliability calibration of bridge design code", Journal of Structural Engineering, Vol. 112, No. 4, pp. 745-763. https://doi.org/10.1061/(asce)0733-9445(1986)112:4(745)

Guenov, M.D. et al. (2018), "Margin Allocation and Tradeoff in Complex Systems Design and Optimization", AIAA Journal, Vol. 56 No. 7, pp. 2887-2902. https://doi.org/10.2514/1.j056357

Iorga, C., Desrochers, A. and Smeesters, C. (2012), "Engineering design from a safety perspective", Proceedings of the Canadian Engineering Education Association Conference. University of Manitoba. June 17-20, 2012. https://doi.org/10.24908/pceea.v0i0.4654

Lebjioui, S. (2018), Investigating and Managing Design Margins throughout the Product Development Process, The Open University.

Mohammed, E.A. et al. (2016), "Design safety margin of a 10,000 TEU container ship through ultimate hull girder load combination analysis", Marine Structures, Vol. 46, pp. 78-101. https://doi.org/10.1016/ j.marstruc.2015.12.003

Morse, E. et al. (2018), "Tolerancing: Managing uncertainty from conceptual design to final product", CIRP Annals - Manufacturing Technology, Vol. 67 No. 2, pp. 695-717. https://doi.org/10.1016/j.cirp.2018.05.009

Tackett, M.W.P., Mattson, C.A. and Ferguson, S.M. (2014), “A Model for Quantifying System Evolvability Based on Excess and Capacity", Journal of Mechanical Design, Vol. 136, pp. 5. https://doi.org/10. 1115/1.4026648

Tan, J., Otto, K. and Wood, K. (2016), "Concept Design Trade-Offs Considering Performance Margins", in Boks, C., Sigurjonsson, J., Steinert, M., Vis, C. and Wulvik, A. (Eds) DS 85-1: Proceedings of NordDesign 2016, Volume 1, Trondheim, Norway, 10th-12th August 2016, p. 421.

Tilstra, A.H. et al. (2015), "Principles for designing products with flexibility for future evolution", International Journal of Mass Customisation, Vol. 5 No. 1, pp. 22-54. https://doi.org/10.1504/ijmassc.2015.069597

Watson, J.D. et al. (2016), “Optimization of excess system capability for increased evolvability”, Structural and Multidisciplinary Optimization, Vol. 53 No. 6, pp. 1277-1294. https://doi.org/10.1007/s00158-015-1378-x

Zhu, J. and Ting, K. (2000), "Performance Distribution Analysis and Robust Design", Journal of Mechanical Design, Vol. 123 No. 1, pp. 11-17. https://doi.org/10.1115/1.1333095 